\title{
Variable-number tandem repeat 3690 polymorphism in Indian clinical isolates of Mycobacterium tuberculosis and its influence on transcription
}

Correspondence

Ranjana Srivastava

drranjana@yahoo.com

Received 12 April 2008

Accepted 8 February 2009

\author{
Parvez Akhtar, ${ }^{1}$ Sarman Singh, ${ }^{2}$ Pablo Bifani, ${ }^{3}$ Satinder Kaur, ${ }^{1}$ \\ Brahm S. Srivastava ${ }^{1}$ and Ranjana Srivastava ${ }^{1}$ \\ ${ }^{1}$ Microbiology Division, Central Drug Research Institute, Lucknow 226001, India \\ ${ }^{2}$ Department of Laboratory Medicine, All India Institute of Medical Sciences, New Delhi, India \\ ${ }^{3}$ Molecular Pathology of Tuberculosis, Pasteur Institute, Brussels, Belgium
}

\begin{abstract}
Variable-number tandem repeat (VNTRs) occur throughout the chromosome of Mycobacterium tuberculosis. Although these polymorphic VNTRs, also known as mycobacterial interspersed repetitive units (MIRUs), have proved to be useful tools in molecular epidemiology, their biological significance is less well understood. This study investigated the polymorphism of the VNTR 3690 locus located in the intergenic region between $r v 3304$ and $r v 3303 c$ (encoding the gp/D2 and $I p d A$ genes, respectively) and its possible function in the regulation of gene expression. The copy number of VNTR 3690 was found to vary among Indian clinical isolates of M. tuberculosis (one to twelve copies), M. tuberculosis H37Rv TMC102 (four copies), M. tuberculosis H37Ra (two to four copies), Mycobacterium bovis BCG (one copy). The expression of IpdA as measured by quantitative RT-PCR was 12 -fold higher in M. tuberculosis H37Rv than in M. bovis BCG. Using a GFP reporter system in which the $5^{\prime}$-flanking region of $I p d A$ was fused to the gfp gene, the effect of VNTRs on gene expression was measured in an M. bovis BCG host background by real-time PCR. Compared with one VNTR repeat, a 12.5-fold upregulation of GFP expression was found with a flanking region containing four VNTR 3690 repeats, indicating that there is a good correlation between VNTR copy number and transcription of IpdA.
\end{abstract}

\section{INTRODUCTION}

The genomes of eukaryotes and prokaryotes are laden with tandem-repeated DNA sequences occurring in several up to thousands of copies, dispersed throughout the genome. Some of these repeats show inter-individual length variability and are designated variable-number tandem repeats (VNTRs). VNTR loci have been of interest because of hypervariability in the number of repeats at each locus and hence have emerged as useful markers to allow individual identification, and tracking of genetic drift and speciation in populations, in both eukaryotes and prokaryotes (Jeffreys et al., 1985; Van Belkum et al., 1998). Contiguous repeats have been characterized in various prokaryotic genomes (Frothingham \& Meeker-O'Connell, 1998; Keim et al., 2000; Le Flèche et al., 2002; Supply et al., 2000; Van Belkum et al., 1997) and although they are found most often in intergenic regions, they can also form part of coding sequences. Microsatellites with short-sequence repeats are more

Abbreviations: EAI, East African-Indian; MIRU, mycobacterial interspersed repetitive unit; SNP, single-nucleotide polymorphism; VNTR, variable-number tandem repeat. common and have been characterized in various prokaryotic genomes in genes encoding products as diverse as microbial surface components recognizing adhesive matrix molecules and specific virulence factors such as LPS-modifying enzymes or adhesions (Van Belkum et al., 1998). Certain types of bacteria are known to use microsatellite-containing genes called contingency genes, which can confer survival advantages on a subset of the bacterial population under changing environmental conditions; for example, in Neisseria gonorrhoeae, they are used as a means of evading the immune system (Van Belkum et al., 1998).

Minisatellite structures composed of $40-100$ bp repetitive units reported in Mycobacterium tuberculosis are VNTRs (Frothingham \& Meeker-O'Connell, 1998), also known as mycobacterial interspersed repetitive units (MIRUs), originally reported in 41 locations throughout the chromosome (Supply et al., 1997). Twelve of these have been shown to display a polymorphism in MIRU copy number among non-related M. tuberculosis isolates (Supply et al., 2000). The number of characterized polymorphic loci has since been expanded, and variations in the number of 
the repeated units have been evaluated in diverse collection of M. tuberculosis isolates (Smittipat et al., 2005; Supply et al., 2006). These VNTRs have been located in both coding and non-coding regions, and the polymorphism resulting from their inclusion within coding regions has been suggested as a source of antigenic variation for evading the immune response to provide bacterial populations with evolutionary flexibility to adapt to unpredictable environmental changes in situations in which classical regulation of gene expression is not sufficient (Skuce et al., 2002; Supply et al., 2000). The presence of VNTRs in intergenic regions has been widely reported in eukaryotes and shown to influence gene expression; however, this role has not been addressed in M. tuberculosis.

The VNTR 3690 locus, described in this paper, is located in the intergenic region between $r v 3303 c$ and $r v 3304$, in the $5^{\prime}$-flanking region of the $l p d A(r v 3303 c)$ gene. LpdA is a flavoprotein disulfide reductase originally annotated as a probable lipoamide dehydrogenase in $M$. tuberculosis (Argyrou et al., 2004). Whilst the laboratory M. tuberculosis $\mathrm{H} 37 \mathrm{Rv}$ strain TMC102 contains four tandem repeats, Mycobacterium bovis BCG has been found to have only one repeat unit. The copy number of VNTR 3690 has been reported to vary extensively, ranging from one to eight copies in one study (Supply et al., 2006) and from one to 22 in another (Le Flèche et al., 2002). As such, the VNTR 3690 locus has been included as one of the polymorphic VNTRs utilized in the 15 and 24 subsets of VNTRs utilized in molecular epidemiological investigations of tuberculosis (Supply et al., 2006). The presence of one imperfect repeat (with two nucleotide differences) present in all isolates and included as one of the copies in molecular typing is noteworthy. Overall, VNTR 3690, also referred to as Mtub39 (Fabre et al., 2004) or allele no. 7, is 58 bp long and has been found to have an allelic diversity of 0.64 (Le Flèche et al., 2002; Supply et al., 2006). Among 65 clonally and epidemiologically related isolates and single-colony cultures, only one variant was identified. The presence and variability of VNTR 3690 in the intergenic region led us to investigate the hypervariability of this VNTR locus in Indian clinical isolates of $M$. tuberculosis and to address the functional role of the repeat variability in transcription by measuring $l p d A$ transcription by quantitative RT-PCR (qRT-PCR) in M. tuberculosis $\mathrm{H} 37 \mathrm{Rv}$ (four copies) and $M$. bovis BCG (one copy), and by fusing intergenic regions containing four and one repeats of VNTR 3690 with a reporter GFP gene $(g f p)$ and assaying for GFP expression.

\section{METHODS}

M. tuberculosis clinical and reference strains and plasmids. Seventeen H37 variants were obtained from the American Type Culture Collection (ATCC), including H37Rv isolates 25618, 27294, 35820-35830, 35837 and H37Ra isolates 25177, 35835 and 35836 (Bifani et al., 2000). Other reference strains included $M$. bovis BCG ATCC 35737 and Mycobacterium microti ATCC 19422. Clinical isolates included CDC1551 (Valway et al., 1998), W17, W1 and W4 (Bifani et al., 1996), AF9 and AF13 belonging to the same H37 strain family (Bifani et al., 1999; Gutacker et al., 2006), strain Erdman belonging to the Haarlem family and an ancestral East African-Indian (EAI) isolate originating from India. Clinical isolates were a kind gift of B. Kreiswirth (Tuberculosis Center, Public Health Research Institute, NJ, USA). All samples were molecularly characterized by RFLP, spoligotyping, MIRU-VNTR analysis, principal genetic grouping and single-nucleotide polymorphism (SNP) analysis, as reported previously (Bifani et al., 1996, 2000; Gutacker et al., 2006; Sreevatsan et al., 1997). The Indian clinical isolates were obtained from the All India Institute of Medical Sciences, New Delhi, India, and identified by biochemical characterization and 16S rRNA gene sequencing. Mycobacteria were grown in Sauton's liquid medium $\left(0.5 \mathrm{~g} \mathrm{KH}_{2} \mathrm{PO}_{4}\right.$, $0.5 \mathrm{~g} \mathrm{MgSO}_{4} .7 \mathrm{H}_{2} \mathrm{O}, 2.0 \mathrm{~g}$ citric acid, $0.05 \mathrm{~g}$ ferric ammonium citrate, $35 \mathrm{ml}$ glycerol, $4.0 \mathrm{~g}$ L-asparagine and $2.5 \mathrm{ml} 20 \%$ Tween 80 in 1 litre distilled water, $\mathrm{pH}$ adjusted to 7), Middlebrook 7H9 medium (Difco) supplemented with ADC $(0.2 \%$ glucose, $0.5 \%$ BSA fraction V, $0.0003 \%$ beef catalase) or solid Middlebrook 7H10 agar (Difco) with OADC enrichment (Becton Dickinson). All cultures were grown at $37{ }^{\circ} \mathrm{C}$.

Plasmid pLL192 is an Escherichia coli-mycobacterium shuttle promoter probe vector containing $g f p$ and kanamycin resistance reporter genes for cloning of mycobacterial promoters (Srivastava et al., 2007b).

PCR amplification, cloning and nucleotide sequencing. For genomic DNA extraction, mycobacteria were cultured in LowensteinJensen medium for 3 weeks. The cells were harvested and chromosomal DNA was extracted as described previously (Akhtar et al., 2006). The intergenic region between rv3304 and rv3303c (coordinates 3689457 to 3690938 on the M. tuberculosis genome; www. sanger.ac.uk) was PCR-amplified from the genomic DNA of $M$. tuberculosis $\mathrm{H} 37 \mathrm{Rv}$, clinical isolates and other mycobacterial strains by using the primers LpdN (5'-CGGGATCCACGGGGGAAAGCTTAGAC-3', forward primer) and LpdC (5'-CGGGATCCAGCCCATAACCTAGCTC-3', reverse primer). The PCR-amplified products were cloned in TOPO TA vector (Invitrogen) and transformed into $E$. coli TOP10 strain (Invitrogen) following the manufacturer's protocol.

For cloning in the pLL192 vector (Srivastava et al., 2007), the intergenic regions were PCR-amplified from M. tuberculosis H37Rv TMC102 and M. bovis BCG using the LpdN and LpdC primers and subcloned into the BamHI site of pLL192. Recombinant plasmids containing inserts in the correct orientation with respect to the $g f p$ gene were selected by HindIII restriction digestion. The constructs pLL192::P1 and pLL192::P4 containing one and four copies of VNTR3690, respectively, were electroporated into M. bovis BCG. The positive control contained the $h s p 60$ promoter cloned in the vector and the negative control consisted of vector alone. The nucleotide sequence of the cloned intergenic regions was confirmed with an Automated Sequence Analyser (Applied Biosystem Genetic Analyser model 310) using the M13 primers supplied in the kit.

RNA isolation from mycobacteria and real-time PCR. $M$. tuberculosis H37Rv, M. bovis BCG, BCG[pLL192::P1] and BCG[pLL192::P4] cells were grown in Middlebrook 7H9 broth containing ADC and $0.05 \%$ Tween 80 to exponential growth phase $\left(1 \times 10^{8}\right.$ c.f.u. $\left.\mathrm{ml}^{-1}\right)$. Total RNA was isolated using a Qiagen Total RNA isolation kit. Bacterial cells were harvested by centrifugation at $6000 \mathrm{~g}$ at $4{ }^{\circ} \mathrm{C}$ for $10 \mathrm{~min}$. The bacterial pellets (containing up to $1 \times 10^{9}$ c.f.u.) were resuspended in $1050 \mu \mathrm{l}$ RLT buffer (Qiagen) containing $0.1 \% \beta$-mercaptoethanol. Cell suspensions were transferred to $2 \mathrm{ml}$ screw-capped tubes containing $0.5 \mathrm{ml}$ glass beads (diameter $0.1 \mathrm{~mm}$ ). The mixture was shaken in a Mini Bead Beater six times for $30 \mathrm{~s}$ at maximum speed ( 5000 r.p.m.) before performing the rest of the extraction. The tubes were centrifuged for $1 \mathrm{~min}$ at $8000 \mathrm{~g}$ and $4{ }^{\circ} \mathrm{C}$. Supernatants were transferred to microcentrifuge tubes, 0.7 vols ethanol was added and the 
supernatant was applied to two RNeasy columns (Qiagen) per sample, according to the manufacturer's instructions.

The purity of the RNA was determined by measuring absorbance at 260 and $280 \mathrm{~nm}$ and quantified by using a Qubit fluorimeter (Invitrogen). For real-time PCR analysis, each RNA sample was treated with Qiagen RNase-free DNase and heat-inactivated according to the manufacturer's instructions. RT-PCR experiments without prior reverse transcription were performed to ensure exclusion of DNA contaminants. RNA (300-400 ng) was used for cDNA synthesis with random hexamer primers by using a RevertAid First-strand cDNA synthesis kit (Fermentas). Internal primers to amplify a region of around $200-250 \mathrm{bp}$ were designed for the lpdA, sigA, gfp and streptomycin resistance (str) genes using DNASTAR primer designing software.

Real-time quantitative PCR for the $l p d A$ and $g f p$ genes was carried out by using a LightCycler RNA Master SYBR Green kit (Roche). sigA and str RNA were used for normalization. The primers used are listed in Table 1 . All primer sets were tested prior to use to ensure that only a single product of the correct size was amplified for each set. Each reaction was repeated three times with three independent RNA samples in a LightCycler 480 II machine. Negative controls consisting of no-template reaction mixtures were run with all reactions. RT-PCR conditions were an initial denaturation step of $10 \mathrm{~min}$, followed by 40 amplification cycles of $10 \mathrm{~s}$ at $95{ }^{\circ} \mathrm{C}, 20 \mathrm{~s}$ at $60{ }^{\circ} \mathrm{C}$ and $30 \mathrm{~s}$ at $72{ }^{\circ} \mathrm{C}$ ). Melting-curve analysis was carried out to confirm the specificity of the amplified product. Each reaction was repeated three times with three independent RNA samples. After baseline correction and determination of threshold settings, calculations were carried out using the $2^{-\Delta \Delta C t}$ method of Livak \& Schmittgen (2001), as PCR efficiencies were found to be similar. Statistical analysis was carried out using PRISM software. The results are expressed as fold induction, which denotes fold increase in expression of the gene.

\section{RESULTS}

\section{VNTR polymorphism in M. tuberculosis, M. bovis BCG and clinical isolates of $M$. tuberculosis}

The intergenic region between $r v 3303 \mathrm{c}$ and $r v 3304$ was PCR-amplified from the genomic DNA of M. tuberculosis reference strains, clinical isolates and $M$. bovis BCG using the LpdN and LpdC primers. Agarose gel analysis of PCR amplicons followed by nucleotide sequencing revealed four tandem repeats of VNTR 3690 in M. tuberculosis H37Rv TMC102 (laboratory strain), one repeat in M. bovis BCG and between one and 12 copies in clinical isolates of $M$. tuberculosis (Fig. 1). The clinical isolates were isolated from tuberculosis patients and characterized as described in Methods. The distribution of repeats in $\mathrm{H} 37 \mathrm{Rv}$ variants obtained from the ATCC is shown in Table 2, including H37Rv isolates 25618, 27294, 35820-35830 and 35837, H37Ra isolates 25177, 35835 and 35836, reference strains M. bovis BCG ATCC 35737 and M. microti ATCC 19422, clinical isolates CDC1551, W17 and W1, AF9 and AF13 belonging to the same cluster as $\mathrm{H} 37 \mathrm{Rv} / \mathrm{Ra}$, and strain Erdman. The strains and their sources are described in Methods. In all samples, PCR-amplified sequences were cloned in the TOPO TA vector followed by nucleotide sequencing. The sequences flanking the $58 \mathrm{bp}$ repeat were identical in all cases. The nucleotide sequences of the

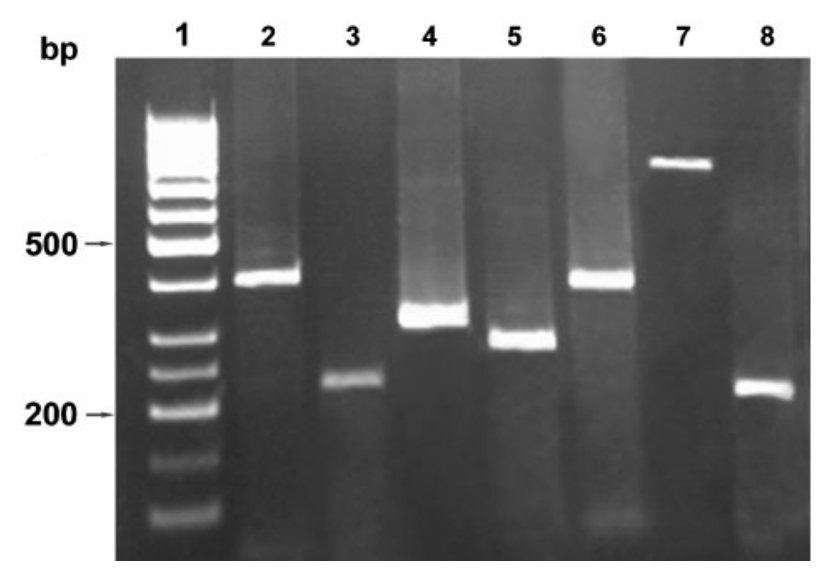

Fig. 1. Agarose (2\%) gel electrophoresis of the PCR-amplified intergenic region from $M$. tuberculosis H37Rv (lane 2), M. bovis BCG (lane 3) and representative Indian M. tuberculosis clinical isolates containing three, two, four, twelve and one copy of VNTR 3690 (lanes 4-8, respectively). Lane 1, 50 bp DNA ladder (MBI Fermentas).

intergenic region between $r v 3303 c$ and $r v 3304$ for $M$. tuberculosis $\mathrm{H} 37 \mathrm{Rv}$ and M. bovis BCG are given in Fig. 2. Variation among the different isolates was only in copy number of VNTR 3690. All samples contained one imperfect repeat $\left(5^{\prime}\right.$-AGCTGTGCTTG ${ }^{\star}$ ACTGCTCGGCTCCTCCTCACGCCGCTGCGCGGCGTGCATCGTCGCCG$3^{\prime}$ ) with two SNPs, followed by a varying number of tandem repeats of VNTR 3690 (Fig. 2). The imperfect repeat carried an extra $\mathrm{C}$ and one $\mathrm{G}$ was missing $\left({ }^{*}\right)$ compared with VNTR 3690. The single copy of this imperfect repeat with an identical sequence was present in the same location in all of the strains and isolates described in Table 2.

\section{Expression of IpdA in M. tuberculosis H37Rv and M. bovis BCG}

The expression of $l p d A$ ( $r v 3303 \mathrm{c}$ ) was monitored in $M$. tuberculosis $\mathrm{H} 37 \mathrm{Rv}$ TMC102 and M. bovis BCG grown in vitro by qRT-PCR using $l p d A$-specific primers (Table 1). RNA was isolated from M. tuberculosis and M. bovis BCG strains grown in vitro and the sigA gene was used as an internal control for normalization of transcriptional analysis. The expression of $l p d A$ in $M$. tuberculosis was found to be 12-fold higher than that observed in M. bovis BCG and this was statistically significant $(P<0.05)$.

\section{Cloning of the intergenic region in pLL192 and the influence of repeat number on transcriptional activity}

As the intergenic region formed the $5^{\prime}$-flanking region of $r v 3303 c$, it was of interest to see whether the region could drive the expression of a heterologous gene cloned downstream from the intergenic region and whether the varying number of tandem repeats influenced 
(a)

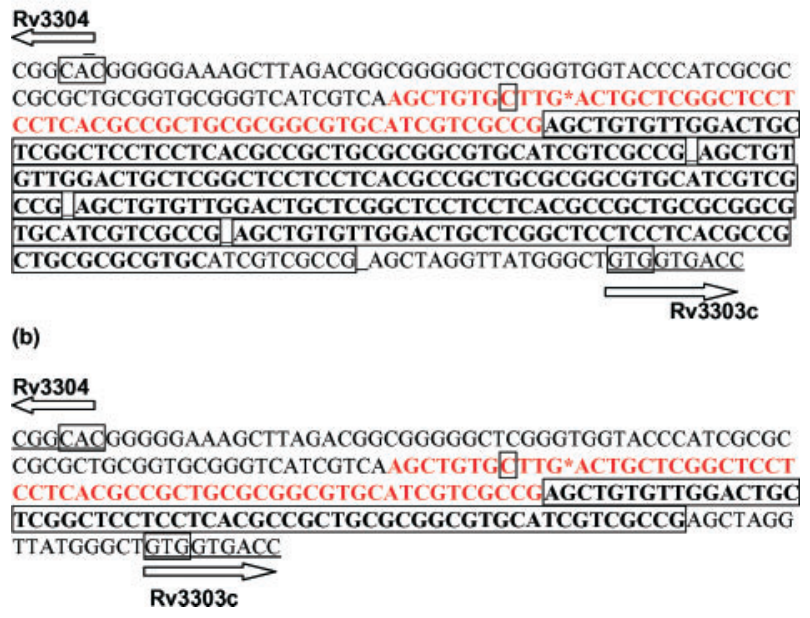

Fig. 2. Nucleotide sequence of the PCR-amplified intergenic region between $r v 3303 c$ and $r v 3304$ from the genomic DNA of $M$. tuberculosis H37Rv TMC102 (378 bp) (a) and M. bovis BCG (204 bp) (b) containing VNTR 3690 and its flanking sequences. The arrows show the location and orientation of the rv3304 and rv3303c genes and their initiation codons (boxed above the arrows) as annotated by Cole et al. (1998). In all samples, VNTR 3690 repeats (boxed) were preceded by an imperfect copy (red) of the repeat containing two SNPs. The imperfect repeat contained an extra $\mathrm{C}$ residue (boxed), and a $\mathrm{G}$ residue $\left(^{*}\right)$ was missing.

transcriptional activity. To check this, two intergenic regions with four and one repeat units were PCR-amplified from their respective genomic DNAs and cloned in the TOPO TA vector. After confirmation of the nucleotide sequence, the inserts were excised from the TOPO vector and subcloned in the BamHI site of the promoter cloning vector pLL192 (Srivastava et al., 2007). pLL192 contains the $g f p$ and kanamycin resistance reporter genes expressed as a transcriptional fusion preceded by a unique $\mathrm{BamHI}$ site, the str gene for selection and the mycobacterial and $E$. coli origins of replication (Srivastava et al., 2007). Transformants with the intergenic region cloned in the correct orientation with respect to the $g f p$ gene were selected and electroporated into $M$. bovis BCG for

Table 1. Primers used for real-time PCR

\begin{tabular}{|c|c|}
\hline Gene & Primer sequence $\left(5^{\prime}-3^{\prime}\right)$ \\
\hline \multirow[t]{2}{*}{$g f p$} & TTGCACTACTGGAAAACTACCTG \\
\hline & TCСАТСТТСТTТААААТСААТАСС \\
\hline \multirow[t]{2}{*}{ str } & TTCAGGAACCGGATCAAAGAGT \\
\hline & GACATCATTCCGTGGCGTTATC \\
\hline \multirow[t]{2}{*}{$\operatorname{lpdA}$} & GCTCAGCATGGGGGTTCAG \\
\hline & CGCGTCCAAGTCGTAGAGC \\
\hline \multirow[t]{2}{*}{$\operatorname{sig} A$} & CCGGGGCATGGCGTTTCTC \\
\hline & ATGCGGCCCAGCTTGTTGAT \\
\hline
\end{tabular}

expression analysis. BCG transformants containing pLL192::P1 (one repeat) or pLL192::P4 (four repeats) were grown to exponential phase and total RNA was isolated for $g f p$ expression analysis by qRT-PCR, using strspecific primers for normalization. Both constructs expressed GFP and were kanamycin resistant, suggesting the presence of a putative promoter in the intergenic region, i.e. 5'-flanking region of the $l p d A$ gene. The expression of $g f p$ in BCG[pLL192:: P4] was 12.5-fold higher than in BCG[pLL192::P1] and this difference was statistically significant (Fig. 3). The differential expression of $g f p$ in the two constructs was not related to plasmid copy number as it was normalized with str (plasmid marker) as an internal control. No difference in $l p d A$ expression was observed by qRT-PCR of $l p d A$ using sigA for normalization (Fig. 3). The results of these qRT-PCR experiments thus demonstrated a direct association between repeat number and transcription of the gene.

\section{DISCUSSION}

Mycobacteria contain highly polymorphic VNTR elements in their genome (Le Flèche et al., 2002; Supply et al., 2006). In this investigation, we characterized the VNTR 3690 polymorphism located in the intergenic region between $r v 3304$ and $r v 3303 c$, the latter having been annotated as lpdA, and analysed its role in the regulation of expression of $1 p d A$. LpdA, encoded by $r v 3303 \mathrm{c}$, appears to be a virulence factor of $M$. tuberculosis. The protein has been characterized as encoding a new member of the flavoprotein disulfide reductase catalysing the $\mathrm{NAD}(\mathrm{P}) \mathrm{H}$-dependent reduction of alternative electron acceptors such as 2,6-dimethyl-1,4-benzoquinone and 5-hydroxy-1,4naphthoquinone (Argyrou et al., 2004), and thus may contribute to the energy requirements under anaerobic conditions. LpdA was one of the major antigens of $M$. tuberculosis identified in clinical sera through the in vivoinduced antigen technology approach (Deb et al., 2002). LpdA has been shown to protect $M$. tuberculosis from oxidative stress in a mouse model and to contribute to enhanced survival of the tubercle bacillus (Akhtar et al., 2006). NADPH quinone reductase in Helicobacter pylori has been shown to play a similar role in oxidative stress and host colonization (Wang \& Maier, 2004). It was intriguing to determine whether the presence of more than one copy of VNTR in the $5^{\prime}$-flanking region of $r v 3303$ c correlated with expression of $l p d A$.

VNTR 3690 is highly polymorphic (Le Flèche et al., 2002; Supply et al., 2006) and exists with variable copy number in M. tuberculosis $\mathrm{H} 37 \mathrm{Rv}$ TMC102 and M. bovis BCG; there are four copies in the former and one in the latter. Determination of the presence of VNTR 3690 was extended to a collection of clinical isolates from northern India and reference strains belonging to the H37Rv/RA family including H37Rv, H37Ra variants, M. bovis BCG, representative $M$. tuberculosis strains of the W/Beijing genotype and CDC1551. These reference strains have been typed 
Table 2. Distribution of VNTR 3690 in M. tuberculosis reference strains, clinical isolates and other mycobacteria ND, Not determined.

\begin{tabular}{|c|c|c|c|c|c|}
\hline $\begin{array}{l}\text { M. tuberculosis } \\
\text { IS6110 fingerprint }\end{array}$ & $\begin{array}{l}\text { Reference } \\
\text { code }^{*}\end{array}$ & No. of repeats & $\begin{array}{l}\text { Strain } \\
\text { family }\end{array}$ & Cluster group $\dagger$ & $\begin{array}{l}\text { Amplicon } \\
\text { size (bp) }\end{array}$ \\
\hline \multicolumn{6}{|l|}{ H37 strains } \\
\hline Rv9 & ATCC 25618 & 4 & H37 reference & VIII & 378 \\
\hline Rv7 & ATCC 27294 & 4 & H37 reference & VIII & 378 \\
\hline Rv1 & ATCC 35820 & 6 & H37 reference & VIII & 494 \\
\hline Rv2 & ATCC 35821 & 3 & H37 reference & VIII & 320 \\
\hline Rv8 & ATCC 35822 & 4 & H37 reference & VIII & 378 \\
\hline Rv3 & ATCC 35823 & 4 & H37 reference & VIII & 378 \\
\hline Rv5 & ATCC 35824 & 3 & H37 reference & VIII & 320 \\
\hline Rv6 & ATCC 35825 & 4 & H37 reference & VIII & 378 \\
\hline Rv8 & ATCC 35826 & 4 & H37 reference & VIII & 378 \\
\hline Rv8 & ATCC 35827 & 4 & H37 reference & VIII & 378 \\
\hline Rv8 & ATCC 35828 & 4 & H37 reference & VIII & 378 \\
\hline Rv4 & ATCC 35829 & 4 & H37 reference & VIII & 378 \\
\hline Rv8 & ATCC 35830 & 4 & H37 reference & VIII & 378 \\
\hline Rv7 & ATCC 35837 & 3 & H37 reference & VIII & 320 \\
\hline Ra1 & ATCC 25177 & 2 & H37 reference & VIII & 262 \\
\hline Ra1 & ATCC 35835 & 4 & H37 reference & VIII & 378 \\
\hline Ra1 & ATCC 35836 & 4 & H37 reference & VIII & 378 \\
\hline H37Rv-25618\$ & TMC 102 & 4 & H37 reference & VIII & 378 \\
\hline AF9 & TN 5705 & 4 & H37 clinical & VIII & 378 \\
\hline AF13 & TN 3095 & 4 & H37 clinical & VIII & 378 \\
\hline \multicolumn{6}{|l|}{ W/Beijing strains } \\
\hline W17 & TN 2015 & 1 & W/Beijing & II & 204 \\
\hline W1 & TN 7759 & 1 & W/Beijing & II & 204 \\
\hline W4 & TN 10775 & 1 & W/Beijing & II & 204 \\
\hline KY & TN 8717 & 1 & W/Beijing & II & 204 \\
\hline \multicolumn{6}{|l|}{ Other strains } \\
\hline HR102 & TN 19546 & 1 & EAI & I & 204 \\
\hline Erdman strain & TMC 107 & 4 & Haarlem & III & 378 \\
\hline CDC1551 & TN 5970 & 2 & $\mathrm{CDC}$ & $\mathrm{V}$ & $260 \|$ \\
\hline $\mathrm{P}$ & TN 1618 & 1 & LAM & VI & 204 \\
\hline \multicolumn{6}{|c|}{ M. tuberculosis-complex strains } \\
\hline M. bovis $\mathrm{BCG}$ & ATCC 35737 & 1 & Reference & I & 204 \\
\hline M. microti & ATCC 19422 & 3 & Reference & I & 320 \\
\hline Mycobacterium africanum & TMC 5122 & 1 & Reference & I & 204 \\
\hline \multicolumn{6}{|c|}{ M. tuberculosis Indian clinical isolates } \\
\hline Six isolates & $\mathrm{ND}$ & 1 & Clinical & ND & 204 \\
\hline 58 isolates & $\mathrm{ND}$ & 2 & Clinical & $\mathrm{ND}$ & 262 \\
\hline 28 isolates & ND & 3 & Clinical & ND & 320 \\
\hline Four isolates & ND & 4 & Clinical & $\mathrm{ND}$ & 378 \\
\hline One isolate & $\mathrm{ND}$ & 12 & Clinical & ND & 842 \\
\hline
\end{tabular}

${ }^{\star}$ TMC, Trudeau Mycobacterial Collection; TN, tracking number, Public Health Research Institute (NJ, USA).

$\dagger$ Genetic cluster according to Gutacker et al. (2006).

¥Includes $146 \mathrm{nt}$ comprising one $58 \mathrm{bp}$ imperfect repeat and flanks. Each additional repeat is 58 bp long.

§Same strain as ATCC 25618 but from a different source.

IITwo bases missing on second repeat.

previously by various molecular techniques including IS6110, principal genetic grouping, genetic clustering (SNPs) and spoligotyping (Bifani et al., 2000). The 17 ATCC H37 variants have been discriminated into nine distinct yet similar patterns (Rv1-Rv9) with a single pattern for all H37Ra (Ra1) isolates. These variants could not be discriminated by polymorphic GC-repetitive sequences and VNTR analysis (Bifani et al., 2000). 


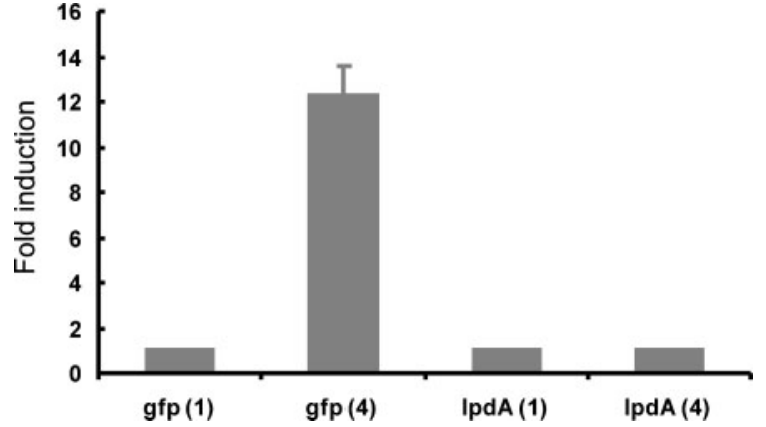

Fig. 3. Transcriptional analysis of gfp expression in BCG[pLL192::P1] and BCG[pLL192::P4] in culture. P1 and $\mathrm{P} 4$ are the intergenic regions between rv3304 and $r v 3303 c$ containing one and four copies, respectively, of VNTR 3690 cloned into the pLL192 vector. Recombinant $M$. bovis BCG was grown to exponential phase and RNA was isolated. The transcriptional levels of $g f p$ and IpdA were determined by qRTPCR, as described in Methods, using str and $\operatorname{sig} A$ as internal controls for normalization, respectively. (1) and (4) represent the copy number of VNTR repeats. The recombinant BCG containing four VNTR repeats showed 12.5-fold enhanced expression of $g f p$ expression compared with rBCG containing one VNTR repeat, whilst there was no difference in $I p d A$ transcription between the constructs. The fold induction observed with four repeats was statistically significant $(P<0.05)$. Error bars, SD.

PCR amplification of genomic DNA of different strains using the LpdN and LpdC primers and subsequent nucleotide sequencing revealed that $13 \mathrm{Rv}$ variants had varying repeat numbers ranging from three to six, with ten strains having four repeats, three having three repeats and one having six repeats. Out of three $\mathrm{Ra}$ strains analysed, two had four repeats, whilst one had two repeats (Table 2). The genome sequence of $M$. tuberculosis sequence published by Cole et al. (1998) (www.sanger.ac.uk) contained only one copy of VNTR 3690, as found in M. bovis BCG (Table 1). The variability encountered in the strains belonging to all three principal genetic groupings (Sreevatsan et al., 1997) suggested that the repeat can be either lost or duplicated. This is particularly evident when examining the $\mathrm{H} 37$ variants. The original H37Rv strain was isolated from a patient at the Trudeau Sanitorium in 1905 and later dissociated into the H37Rv (virulent) and $\mathrm{H} 37 \mathrm{Ra}$ (attenuated) strains in 1934 (Bifani et al., 2000; Steenken \& Gardner, 1946). All other variants evolved from these two original isolates. As such, the data suggest that both Rv and Ra strains possessed a 378 bp fragment (four repeats) at the time of dissociation, as several of its descendents still have four copies ( $\mathrm{Rv} 35822$, 35823, 35825, 35826, 35827, 35828, 35829, 35830, 25618 and 27294, and Ra 35835 and 35836). This observation is further supported by analysis of the recently isolated clinical isolates AF9 and AF13, which share the $378 \mathrm{bp}$ amplicon (Table 2). Only isolate 35820 was found to have duplication, whilst others had deletions (Rv 35821, 35824 and 35837, and Ra 25177). However, analysis of the Indian clinical isolates revealed a different picture. Only four isolates shared the $378 \mathrm{bp}$ fragment (four repeats), 28 isolates had three repeats (320 bp), 58 had two repeats $(262 \mathrm{bp})$, six had one repeat and one had 12 repeats $(842 \mathrm{bp})$. The clinical isolates were from patients belonging to different regions. If the original isolates had four repeats, then most have undergone deletion with only one having undergone duplication. Further analysis of repeat variability in clinical isolates from different geographical regions will provide an insight into strain polymorphism related to this VNTR locus.

The dispersal of repetitive sequences throughout the genome indicates a possible functional role which is yet to be explored. The location of VNTR loci in promoter regions and their influence on gene activity has been reported in higher eukaryotes. Some examples include VNTR polymorphism in the promoter region of the thiopurine methyltransferase gene (Spire-Vayron de la Moureyre et al., 1999), the human insulin 5'-flanking region influencing tissue-specific expression of insulin and insulin-like growth factor (Eerligh et al., 2004) and the 5' upstream promoter region of the prostacyclin synthase gene affecting transcriptional activity (Amano et al., 2004). In the case of prokaryotes, in M. tuberculosis, VNTR loci have been found located both in the intergenic regions and within genes (Supply et al., 2000, 2001). VNTR 3690 is located in the $5^{\prime}$-flanking region of the $\operatorname{lp} d A$ gene, which led us to assess the role of repeats in modulating the expression of $l p d A$. The first evidence was derived from transcriptional analysis of the $l p d A$ gene in M. tuberculosis and $M$. bovis BCG by qRT-PCR. The results demonstrated $\sim 12$-fold enhancement of expression in M. tuberculosis with four repeats compared with $M$. bovis BCG with one repeat. These results are supported by recent reports of a differential expression phenotype of $l p d A$ transcription in M. tuberculosis $\mathrm{H} 37 \mathrm{Rv}$ and H37Ra strains as determined by qRT-PCR (Zheng et al., 2008), where the 58 bp tandem repeat unit of VNTR 3690 was repeated twice in $M$. tuberculosis $\mathrm{H} 37 \mathrm{Rv}$ but three times in $M$. tuberculosis H37Ra, and in exponential-phase cultures, where the transcription of $l p d A$ in H37Ra was $~ 3$-fold higher than in $\mathrm{H} 37 \mathrm{Rv}$.

The intergenic region between $l p d A(r v 3303 c)$ and $r v 3304$, when cloned in the promoter probe vector pLL192 (Saxena et al., 2008; Srivastava et al., 2007, 2008), drove the expression of both $g f p$ and kanamycin resistance reporter genes, indicating the presence of a putative promoter, and variable repeat number was found to influence the level of gfp expression. $M$. bovis BCG harbouring pLL192 with a cloned intergenic region containing one or four copies of the VNTR showed differences in GFP fluorescence and kanamycin resistance. These results allow us to conclude that VNTR copy number has an effect on transcription of $l p d A$ and suggest the presence of a putative promoter within the intergenic region. A hairpin structure (CACGCCGCTGCGCGGCGTG) present within the sequence of the repeat unit has been suggested as a 
potential binding site for regulatory proteins (Zheng et al., 2008). Regulation of promoter activity through VNTRs may be a way of using transcriptional regulation to adapt to the environmental changes to which $M$. tuberculosis is exposed both outside and inside its human host.

\section{ACKNOWLEDGEMENTS}

Thanks are due to the Director, Central Drug Research Institute, for facilities and to CSIR for SRF to P.A. and S.K. This is communication number 7491 of this institute.

\section{REFERENCES}

Akhtar, P., Srivastava, S., Srivastava, A., Srivastava, M., Srivastava, B. S. \& Srivastava, R. (2006). Rv3303c of Mycobacterium tuberculosis protects tubercle bacilli against oxidative stress in vivo and contributes to virulence in mice. Microbes Infect 8, 2855-2862.

Amano, S., Tatsumi, K., Tanabe, N., Kasahara, Y., Kurosu, K., Takiguchi, Y., Kasuya, Y., Kimura, S. \& Kuriyama, T. (2004). Polymorphism of the promoter region of prostacyclin synthase gene in chronic thromboembolic pulmonary hypertension. Respirology $\mathbf{9}$, 184-189.

Argyrou, A., Vetting, M. W. \& Blanchard, J. S. (2004). Characterization of a new member of the flavoprotein disulfide reductase family of enzymes from Mycobacterium tuberculosis. J Biol Chem 279, 5269452702.

Bifani, P. J., Plikaytis, B. B., Kapur, V., Stockbauer, K., Pan, X., Lutfey, M. L., Moghazeh, S. L., Eisner, W., Daniel, T. M. \& other authors (1996). Origin and interstate spread of a New York City multidrugresistant Mycobacterium tuberculosis clone family. JAMA 275, 452457.

Bifani, P. J., Mathema, B., Liu, Z., Moghazeh, S. L., Shopsin, B., Tempalski, B., Driscol, J., Frothingham, R., Musser, J. M. \& other authors (1999). Identification of a $W$ variant outbreak of Mycobacterium tuberculosis via population-based molecular epidemiology. JAMA 282, 2321-2327.

Bifani, P., Moghazeh, S., Shopsin, B., Driscoll, J., Ravikovitch, A. \& Kreiswirth, B. N. (2000). Molecular characterization of Mycobacterium tuberculosis $\mathrm{H} 37 \mathrm{Rv} / \mathrm{Ra}$ variants: distinguishing the mycobacterial laboratory strain. J Clin Microbiol 38, 3200-3204.

Cole, S. T., Brosch, R., Parkhill, J., Garnier, T., Churcher, C., Harris, D., Gordon, S. V., Eiglmeier, K., Gas, S. \& other authors (1998). Deciphering the biology of Mycobacterium tuberculosis from the complete genome sequence. Nature 393, 537-544.

Deb, D. K., Dahiya, P., Srivastava, K. K., Srivastava, R. \& Srivastava, B. S. (2002). Selective identification of new therapeutic targets of Mycobacterium tuberculosis by IVIAT approach. Tuberculosis (Edinb) 82, 175-182.

Eerligh, P., Roep, B. O., Giphart, M. J. \& Koeleman, B. P. C. (2004). Insulin-like growth factor 1 promoter polymorphism influences insulin gene variable number of tandem repeat-associated risk for juvenile onset type 1 diabetes. Tissue Antigens 63, 568-571.

Fabre, M., Koeck, J. L., Le Fleche, P., Simon, F., Herve, V., Vergnaud, G. \& Pourcel, C. (2004). High genetic diversity revealed by variablenumber tandem repeat genotyping and analysis of hsp65 gene polymorphism in a large collection of "Mycobacterium canettii" strains indicates that the Mycobacterium tuberculosis complex is a recently emerged clone of " $M$. canettii". J Clin Microbiol 42, $3248-3255$.
Frothingham, R. \& Meeker-O'Connell, W. A. (1998). Genetic diversity in the Mycobacterium tuberculosis complex based on variable number of tandem DNA repeats. Microbiology 144, 1189-1196.

Gutacker, M. M., Mathema, B., Soini, H., Shashkine, E., Kreiswirth, B. N., Graviss, E. A. \& Musser, J. M. (2006). Single-nucleotide polymorphism-based population genetic analysis of Mycobacterium tuberculosis strains from 4 geographical sites. J Infect Dis 193, 121-128.

Jeffreys, A. J., Wilson, V. \& Thein, S. L. (1985). Hypervariable minisatellite regions in human DNA. Nature 314, 67-73.

Keim, P., Price, L. B., Klevytska, A. M., Smith, K. L., Schupp, J. M., Okinaka, R., Jackson, P. J. \& Hugh-Jones, M. E. (2000). Multiplelocus variable-number tandem repeat analysis reveals genetic relationships within Bacillus anthracis. J Bacteriol 182, 2928-2936.

Le Flèche, P., Fabre, M., Denoeud, F., Koeck, J. \& Vergnaud, G. (2002). High resolution, on-line identification of strains from the Mycobacterium tuberculosis complex based on tandem repeat typing. BMC Microbiol 2, 37-49.

Livak, K. J. \& Schmittgen, T. D. (2001). Analysis of relative gene expression data using real-time quantitative PCR and the $2^{-\Delta \Delta \mathrm{Ct}}$ method. Methods 25, 402-408.

Saxena, A., Srivasrtava, V., Srivastava, R. \& Srivastava, B. S. (2008). Identification of genes of Mycobacterium tuberculosis upregulated during anaerobic persistence by fluorescence and kanamycin resistance selection. Tuberculosis (Edinb) 88, 518-525.

Skuce, R. A., McCorry, T. P., McCarroll, J. F., Roring, S. M. M., Scott, A. N., Brittain, D., Hughes, S. L., Hewinson, R. G. \& Neill, S. D. (2002). Discrimination of Mycobacterium tuberculosis complex bacteria using novel VNTR-PCR targets. Microbiology 148, 519-528.

Smittipat, N., Billamas, P., Palittapongarnpim, M., Thong-on, A., Temu, M. M., Thanakijcharoen, P., Karnkawinpong, O. \& Palittapongarnpim, P. (2005). Polymorphism of variable number tandem repeats at multiple loci in Mycobacterium tuberculosis. J Clin Microbiol 43, 5034-5043.

Spire-Vayron de la Moureyre, C., Debuysère, H., Fazio, F., Sergent, E., Bernard, C., Sabbagh, N., Marez, D., Lo Guidice, J. M., D'halluin, J. C. \& Broly, F. (1999). Characterization of a variable number tandem repeat region in the thiopurine $S$-methyltransferase gene promoter. Pharmacogenetics 9, 189-198.

Sreevatsan, S., Pan, X., Stockbauer, K. E., Connell, N. D., Kreiswirth, B. N., Whittam, T. S. \& Musser, J. M. (1997). Restricted structural gene polymorphism in the Mycobacterium tuberculosis complex indicates evolutionarily recent global dissemination. Proc Natl Acad Sci U S A 94, 9869-9874.

Srivastava, V., Rouanet, C., Srivastava, R., Ramalingam, B., Locht, C. \& Srivastava, B. S. (2007). Macrophage-specific M. tuberculosis genes identified by green fluorescent protein and kanamycin resistance selection. Microbiology 153, 659-666.

Srivastava, V., Jain, A., Srivastava, B. S. \& Srivastava, R. (2008). Selection of genes of Mycobacterium tuberculosis upregulated during residence in lungs of infected mice. Tuberculosis (Edinb) 88, 171-177.

Steenken, W., Jr \& Gardner, L. U. (1946). History of H37 strain of tubercle bacillus. Am Rev Tuberc 54, 62-66.

Supply, P., Magdalena, J., Himpens, S. \& Locht, C. (1997). Identification of novel intergenic repetitive units in a mycobacterial two-component system operon. Mol Microbiol 26, 991-1003.

Supply, P., Mazars, E., Lesjean, S., Vincent, V., Gicquel, B. \& Locht, C. (2000). Variable human minisatellite-like regions in the Mycobacterium tuberculosis genome. Mol Microbiol 36, 762-771.

Supply, P., Lesjean, S., Savine, E., Kremer, L., Van Soolingen, D. \& Locht, C. (2001). Automated high-throughput genotyping for study of global epidemiology of Mycobacterium tuberculosis based on mycobacterial interspersed repetitive units. J Clin Microbiol 39, 3563-3571. 
Supply, P., Allix, C., Lesjean, S., Cardoso-Oelemann, M., RüschGerdes, S., Willery, E., Savine, E., de Haas, P., van Deutekom, H. \& other authors (2006). Proposal for standardization of optimized mycobacterial interspersed repetitive unit-variable-number tandem repeat typing of Mycobacterium tuberculosis. J Clin Microbiol 44, 4498-4510.

Valway, S. E., Sanchez, M. P., Shinnick, T. F., Orme, I., Agerton, T., Hoy, D., Jones, J. S., Westmoreland, H. \& Onorato, I. M. (1998). An outbreak involving extensive transmission of a virulent strain of Mycobacterium tuberculosis. N Engl J Med 338, 633-639.

Van Belkum, A., Scherer, S., van Leeuwen, W., Willemse, D., van Alphen, L. \& Verbrugh, H. (1997). Variable number of tandem repeats in clinical strains of Haemophilus influenzae. Infect Immun 65, 5017-5027.

Van Belkum, A., Scherer, S., Van Alphen, L. \& Verbrugh, H. (1998). Short-sequence repeats in prokaryotic genomes. Microbiol Mol Biol Rev 62, 275-293.

Wang, G. \& Maier, R. J. (2004). An NADPH quinone reductase of Helicobacter pylori plays an important role in oxidative stress resistance and host colonization. Infect Immun 72, 1391-1396.

Zheng, H., Lu, L., Wang, B., Pu, S., Zhang, X., Zhu, G., Shi, W., Zhang, L., Wang, H. \& other authors (2008). Genetic basis of virulence attenuation revealed by comparative genomic analysis of Mycobacterium tuberculosis strain H37Ra versus H37Rv. PLoS One 3, e2375. 